\title{
Urinary interleukin-18 does not predict acute kidney injury after adult cardiac surgery: a prospective observational cohort study
}

\author{
Michael Haase ${ }^{1,2}$, Rinaldo Bellomo ${ }^{1}$, David Story ${ }^{3}$, Piers Davenport ${ }^{4}$ and Anja Haase-Fielitz ${ }^{1,2}$ \\ 1Department of Intensive Care, Austin Health, Melbourne, 145 Studley Rd, Heidelberg 3084, Australia \\ ${ }^{2}$ Department of Nephrology and Intensive Care Medicine, Charité University Medicine, 1 Augustenburger Platz, Berlin 13353 Germany \\ ${ }^{3}$ Department of Anaesthesiology, Austin Health, Melbourne, 145 Studley Rd, Heidelberg 3084, Australia \\ ${ }^{4}$ Department of Medicine, Monash Medical Centre, 168 Clayton Rd, Clayton 3168, Melbourne, Australia \\ Corresponding author: Rinaldo Bellomo, rinaldo.bellomo@med.monash.edu.au
}

Received: 19 May 2008 Revisions requested: 7 Jul 2008 Revisions received: 16 Jul 2008 Accepted: 1 Aug 2008 Published: 1 Aug 2008

Critical Care 2008, 12:R96 (doi:10.1186/cc6972)

This article is online at: http://ccforum.com/content/12/4/R96

(c) 2008 Haase et al.; licensee BioMed Central Ltd.

This is an open access article distributed under the terms of the Creative Commons Attribution License (http://creativecommons.org/licenses/by/2.0), which permits unrestricted use, distribution, and reproduction in any medium, provided the original work is properly cited.

\begin{abstract}
Introduction Urinary interleukin-18 (IL-18) measured during the immediate postoperative period could be a promising predictor of acute kidney injury following adult cardiac surgery.

Methods In a single-centre prospective observational cohort study, we enrolled 100 adult cardiac surgical patients undergoing cardiopulmonary bypass at a tertiary hospital. We measured the urinary concentration of $\mathrm{IL}-18$ and creatinine preoperatively, on arrival in the intensive care unit, and 24 hours postoperatively. We assessed urinary IL-18 concentration and urinary IL-18/urinary creatinine ratio in relation to the postoperative development of acute kidney injury defined as an increase in serum creatinine of greater than $50 \%$ from preoperative to postoperative peak value within 48 hours after surgery.
\end{abstract}

Results Twenty patients developed acute kidney injury. On arrival in the intensive care unit and at 24 hours postoperatively, urinary IL-18 (median [interquartile range]) was not different in patients who subsequently developed acute kidney injury compared with those who did not: on arrival in the intensive care unit (168 [717] versus 104 [256] pg/mL; $P=0.70)$ and at 24 hours (195 [483] versus 165 [246] pg/mL; $P=0.47$ ). On arrival in the intensive care unit (area under the curve for the receiver operating characteristic curve [AUC-ROCC] 0.53, 95\% confidence interval $[\mathrm{Cl}] 0.38$ to $0.68 ; P=0.70)$ and at 24 hours postoperatively (AUC-ROCC $0.55,95 \% \mathrm{Cl} 0.40$ to $0.71 ; P=$ 0.48 ), urinary $\mathrm{IL}-18$ was not better than chance in predicting acute kidney injury. All findings were confirmed when urinary IL18 was adjusted for urinary creatinine. Urinary IL-18 correlated with duration of cardiopulmonary bypass $(P<0.001)$.

Conclusion In adults, early postoperative measurement of urinary IL-18 appears not to be valuable in identifying patients who develop acute kidney injury after cardiac surgery, but rather represents a nonspecific marker of cardiopulmonary bypassassociated systemic inflammation.

\section{Introduction}

Acute kidney injury (AKI) is a serious and frequent complication of cardiac surgery [1-3]. Cardiac surgery is the second most frequent cause of $\mathrm{AKI}$ worldwide among critically ill patients [2]. There is a need for early and specific renal biomarkers that would enable the early prediction of $\mathrm{AKI}$ and timely intervention [4]. There is evidence that urinary interleukin-18 (IL-18) - a proinflammatory cytokine released in response to injury to renal tubular epithelial cells and demonstrated in activated macrophages - might act as an earlier biomarker than serum creatinine in predicting $\mathrm{AKI}$ in critically ill adult patients [5-8]. However, in the prediction of contrastinduced nephropathy in adult patients, the value of urinary IL18 is controversial $[9,10]$. Recently, urinary IL-18 was reported to predict $\mathrm{AKI}$ in patients receiving a kidney transplant [11], in critically ill children [12], and in paediatric cardiac surgery [13]. Despite such a promising observation in children, the predictive performance of the measurement of urinary IL-18 immediately after surgery has not yet been assessed in adult cardiac surgical patients undergoing cardiopulmonary bypass

$\overline{\mathrm{AKI}}=$ acute kidney injury; AUC-ROCC = area under the curve for the receiver operating characteristic curve; CPB = cardiopulmonary bypass; ICU $=$ intensive care unit; IL-18 = interleukin-18; RIFLE = Risk of renal dysfunction, Injury to the kidney, Failure of kidney function, Loss of kidney function, and End-stage kidney disease. 
(CPB), the majority of patients receiving cardiac surgery. Accordingly, in this prospective observational cohort study, we sought to investigate whether urinary IL-18 could serve as an early predictive renal biomarker in adult patients undergoing cardiac surgery. We hypothesised that urinary IL-18 measured during the immediate postoperative period would be a promising predictor of AKI following adult cardiac surgery.

\section{Materials and methods Patient population}

In a prospective observational cohort study, we screened 114 adult patients who underwent cardiac surgery necessitating the use of CPB at a tertiary hospital. We excluded patients undergoing emergency operations (time between hospital admission to operation of less than 24 hours) or an operation without the use of $\mathrm{CPB}$, patients with end-stage renal disease (serum creatinine of greater than $300 \mu \mathrm{mol} / \mathrm{L}$ ), kidney transplant patients, and patients under the age of 18 . The local institutional review board of the Austin Hospital, Melbourne, approved this investigation and written informed consent was obtained. This study was in compliance with the Declaration of Helsinki. Clinical practice was not changed or modified for the purpose of the study.

\section{Outcomes}

In this study, we aimed to examine the predictive performance of urinary IL-18 concentration and of urinary IL-18/urinary creatinine ratio on arrival in the intensive care unit (ICU) and at 24 hours after commencement of CPB for the prediction of $\mathrm{AKI}$ defined as an increase in serum creatinine of greater than $50 \%$ within 48 hours postoperatively (Figures 1, 2, 3, 4). Additional renal outcome measures included $\mathrm{AKI}$ defined as a greater than $25 \%$ increase in creatinine, changes in creatinine to 72 hours or 5 days, sustained increase of greater than $50 \%$, sustained increase of greater than $25 \%$, or AKI graded according to a variety of RIFLE (Risk of renal dysfunction, Injury to the kidney, Failure of kidney function, Loss of kidney function, and End-stage kidney disease) criteria [14]. We defined 'sustained' as at least two consecutive significantly increased serum creatinine values. In virtually all patients, sampling at 6 hours after commencement of CPB closely corresponded to arrival of the patient in the ICU. We refer to this time point of sampling as 'arrival in ICU'.

\section{Sampling and measurement of renal function markers}

As part of the study protocol, for the measurement of urinary IL-18 and urinary creatinine, we obtained urine preoperatively (immediately before induction of anaesthesia), on arrival in ICU, and at 24 hours after commencement of CPB. Samples were spun at $1,500 \mathrm{~g}$ for 10 minutes, and the supernatants were stored in equal volumes within 10 minutes at $-80^{\circ} \mathrm{C}$. We adjusted urinary IL-18 concentration for urinary creatinine concentration (urinary IL-18/urinary creatinine ratio). For the measurement of serum creatinine, we collected blood samples preoperatively and at 24,48 , and 72 hours after commence-
Figure 1

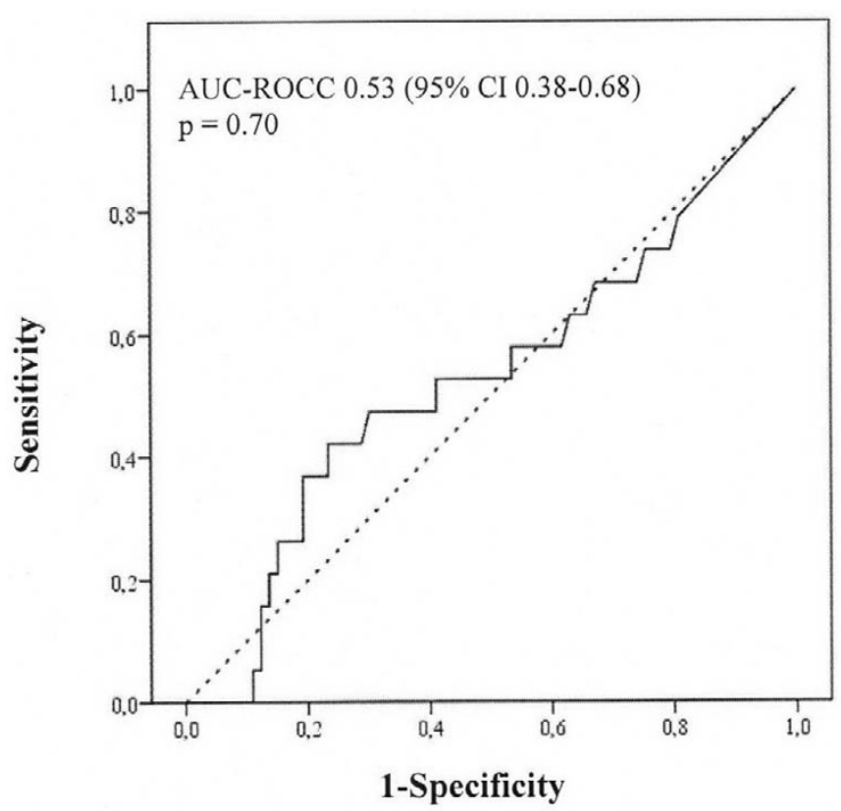

Performance of urinary interleukin-18 on arrival in the intensive care unit as a predictor of acute kidney injury according to an increase in serum creatinine of greater than $50 \%$ from preoperative to postoperative peak value within 48 hours after commencement of cardiopulmonary bypass $(n=20)$. AUCROCC, area under the curve for the receiver operating characteristic curve; $\mathrm{Cl}$, confidence interval.

\section{Figure 2}

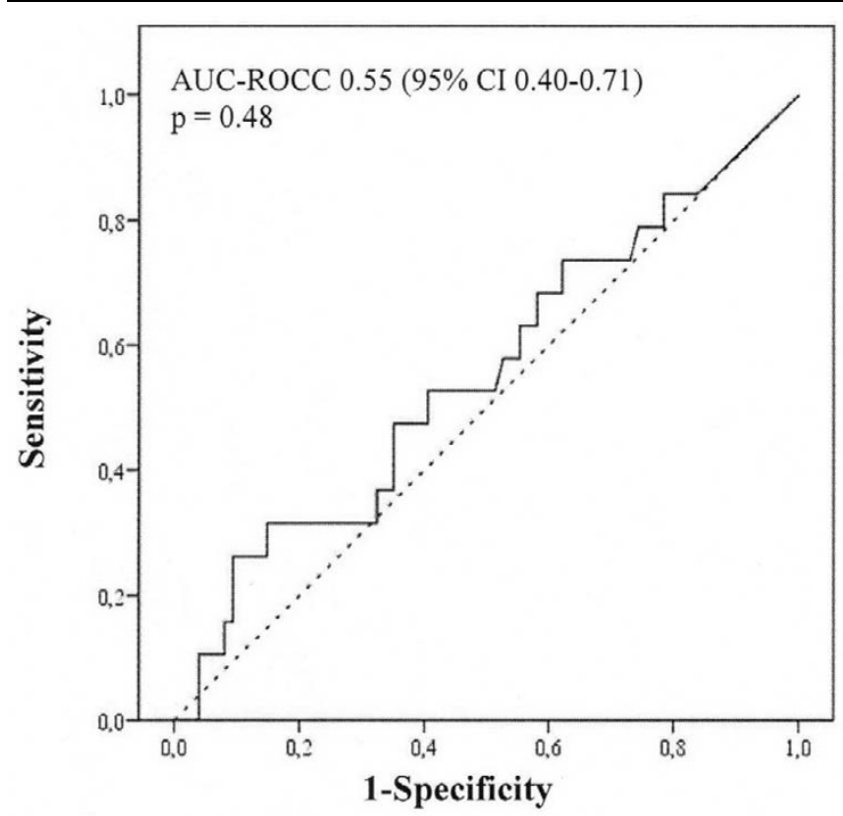

Performance of urinary interleukin-18 at 24 hours after commencement of cardiopulmonary bypass as a predictor of acute kidney injury according to an increase in serum creatinine of greater than $50 \%$ from preoperative to postoperative peak value within 48 hours after commencement of cardiopulmonary bypass $(n=20)$. AUC-ROCC, area under the curve for the receiver operating characteristic curve; $\mathrm{Cl}$, confidence interval. 
Figure 3

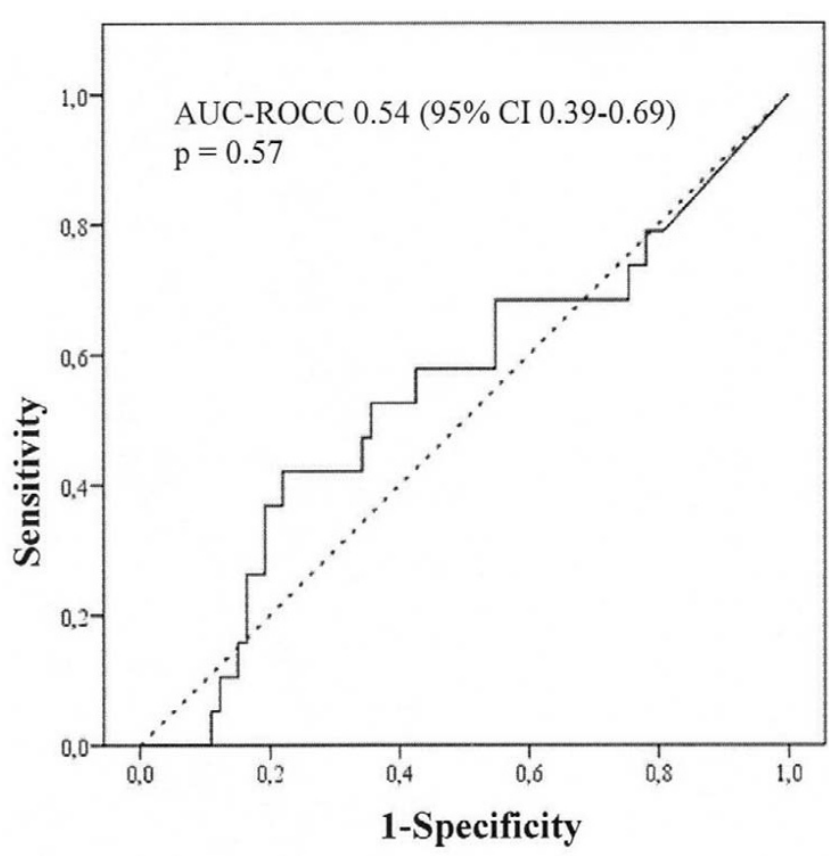

Performance of urinary interleukin-18/urinary creatinine ratio on arrival in the intensive care unit as a predictor of acute kidney injury according to an increase in serum creatinine of greater than $50 \%$ from preoperative to postoperative peak value within 48 hours after commencement of cardiopulmonary bypass $(n=20)$. AUC-ROCC, area under the curve for the receiver operating characteristic curve; $\mathrm{Cl}$, confidence interval.

Figure 4

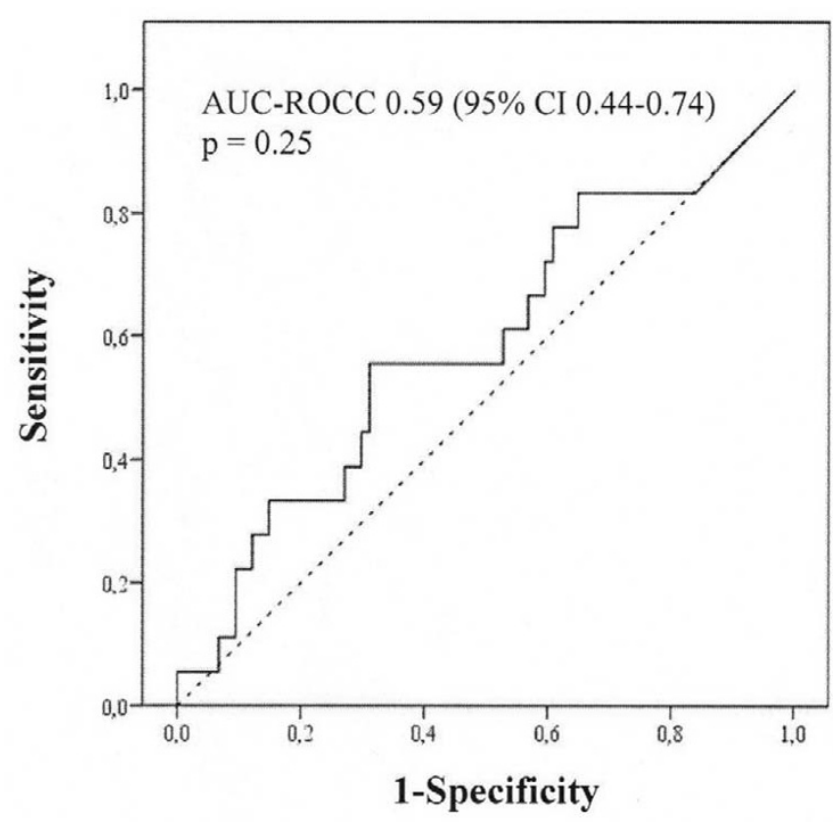

Performance of urinary interleukin-18/urinary creatinine ratio at 24 hours after commencement of cardiopulmonary bypass as a predictor of acute kidney injury according to an increase in serum creatinine of greater than $50 \%$ from preoperative to postoperative peak value within 48 hours after commencement of cardiopulmonary bypass $(n=20)$. AUC-ROCC, area under the curve for the receiver operating characteristic curve; $\mathrm{Cl}$, confidence interval. ment of CPB. Urinary IL-18 was measured in duplicate by enzyme-linked immunosorbent assay using matched antibody pairs and recombinant standards (MBL International, Woburn, MA, USA). The inter- and intra-assay coefficients of variation of urinary IL-18 measurement were less than $8 \%$. Serum and urinary creatinine was measured using the Jaffé method (Beckman Coulter SYNCHRON LX System; Beckman Coulter, Inc., Fullerton, CA, USA). The laboratory investigators were blinded to the sample sources and clinical outcomes.

\section{Data collection}

Data collected preoperatively included age, gender, and information on major comorbidities. We collected intraoperative data on type of cardiac surgery and duration of CPB. Finally, we recorded hourly fluid intake and urinary output from start to 24 hours after commencement of CPB (as a urinary catheter was used in all patients during this time). Urinary output was maintained at 0.5 to $1 \mathrm{~mL} / \mathrm{kg}$ per hour by fluid administration and by using furosemide if necessary.

\section{Statistical analysis}

We assessed data for normal distribution using histograms. When data were normally distributed, we present variables as mean \pm standard deviation and used the $t$ test for comparison of patients developing AKI with those who did not. When data were not normally distributed, we present variables as median with 25th to 75th percentiles and used the Mann-Whitney $U$ test for comparison of both patient groups. When multiple testing occurred, we applied Bonferroni correction. For categorical data, we performed two group comparisons using the chi-square test or Fisher exact test when the expected value was less than 5 . Urinary IL-18 was assessed for its ability to detect $\mathrm{AKI}$ as defined by nonparametric calculation of the area under the curve for the receiver operating characteristic curve (AUC-ROCC) on arrival in ICU and at 24 hours after commencement of CPB. An AUC-ROCC value of 0.90 to 1.0 indicates excellent, 0.80 to 0.89 good, 0.70 to 0.79 fair, 0.60 to 0.69 poor, and 0.50 to 0.59 no useful performance. Urinary IL18 concentration was correlated with duration of CPB (Spearman correlation). A two-sided $P$ value of 0.05 was considered to be statistically significant. We used SPSS version 15.0 (SPSS Inc., Chicago, IL, USA) and MedCalc version 9.3.9.0 (MedCalc Software, Mariakerke, Belgium).

\section{Results}

We excluded 14 patients and analysed data from 100 adult cardiac surgical patients. None of the patients enrolled received contrast dye within 72 hours preoperatively. Arrival in the ICU was $5.5 \pm 1.9$ hours after commencement of CPB. Patient characteristics are presented in Table 1. Twenty patients developed AKI. Patients developing AKI were older and were more likely to present with chronic obstructive pulmonary disease, peripheral vascular disease, atrial fibrillation, and arterial hypertension. Within 48 hours postoperatively, the peak serum creatinine concentration was $182.3 \pm 56.3 \mu \mathrm{mol} /$ 
Table 1

Preoperative characteristics of and intraoperative interventions in patients

\begin{tabular}{|c|c|c|c|}
\hline Characteristics & $A K I(n=20)$ & No AKI $(n=80)$ & $P$ value \\
\hline \multicolumn{4}{|l|}{ Demographic data } \\
\hline Age, years & $75.3 \pm 4.1$ & $68.1 \pm 9.7$ & $<0.001$ \\
\hline Female, number (percentage) & $6(30.0)$ & $33(41.0)$ & 0.36 \\
\hline Body mass index & $29.7 \pm 4.3$ & $27.4 \pm 4.5$ & 0.045 \\
\hline \multicolumn{4}{|l|}{ Comorbidities } \\
\hline Preoperative serum creatinine, $\mu \mathrm{mol} / \mathrm{L}$ & $96.1 \pm 31.6$ & $89.4 \pm 24.0$ & 0.30 \\
\hline Preoperative estimated glomerular filtration rate $<60 \mathrm{~mL} /$ minute, number (percentage) & $8(40.0)$ & $19(23.8)$ & 0.14 \\
\hline Left ventricular dysfunction (ejection fraction < 40\%), number (percentage) & $7(35.0)$ & $18(22.5)$ & 0.25 \\
\hline Arterial hypertension, number (percentage) & $20(100.0)$ & $64(80.0)$ & 0.029 \\
\hline Hypercholesterolemia, number (percentage) & $16(80.0)$ & $46(57.5)$ & 0.06 \\
\hline Atrial fibrillation, number (percentage) & $10(50.0)$ & $16(20.0)$ & 0.006 \\
\hline Myocardial infarction (within 6 months preoperatively), number (percentage) & $7(35.0)$ & $13(16.3)$ & 0.06 \\
\hline Chronic obstructive pulmonary disease, number (percentage) & $6(30.0)$ & $8(10.0)$ & 0.021 \\
\hline Peripheral vascular disease, number (percentage) & $4(20.0)$ & $2(2.5)$ & 0.013 \\
\hline Diabetes mellitus, number (percentage) ${ }^{a}$ & $8(40.0)$ & $20(25.0)$ & 0.18 \\
\hline \multicolumn{4}{|l|}{ Intervention } \\
\hline Coronary artery bypass grafting surgery, number (percentage) & $7(35.0)$ & $18(22.5)$ & 0.25 \\
\hline Valvular surgery, number (percentage) & $7(35.0)$ & $45(56.3)$ & 0.09 \\
\hline Simultaneous coronary revascularisation and valve surgery, number (percentage) & $6(30.0)$ & $11(13.8)$ & 0.08 \\
\hline Duration of cardiopulmonary bypass (minutes) & $147.0 \pm 31.6$ & $136.0 \pm 41.0$ & 0.20 \\
\hline Fluid intake, $0-24$ hours, $\mathrm{mL}^{\mathrm{b}}$ & $5,500 \pm 1,900$ & $5,150 \pm 1,250$ & 0.44 \\
\hline Urine output, $0-24$ hours, $\mathrm{mL}$ & $3,350 \pm 1,050$ & $3,990 \pm 1,500$ & 0.034 \\
\hline Furosemide dose, $0-24$ hours, $\mathrm{mg}$ & $133.9 \pm 168.7$ & $30.9 \pm 34.8$ & $<0.001$ \\
\hline \multicolumn{4}{|l|}{ Outcomes } \\
\hline Need for renal replacement therapy or died during hospital stay, number (percentage) & $4(20.0)$ & $1(1.3)$ & 0.005 \\
\hline Ventilation, days & $2.4 \pm 3.1$ & $0.9 \pm 0.8$ & 0.010 \\
\hline Length of stay in the intensive care unit, days & $6.3 \pm 7.1$ & $2.3 \pm 2.1$ & 0.012 \\
\hline Length of stay in hospital, days & $16.2 \pm 13.8$ & $9.5 \pm 4.7$ & 0.026 \\
\hline
\end{tabular}

aDiabetes mellitus includes patients on antidiabetic medication and on insulin. ${ }^{\mathrm{b}}$ Crystalloids, colloids, and oral fluids from the commencement of cardiopulmonary bypass to 24 hours thereafter. AKI, acute kidney injury.

$\mathrm{L}$ in those patients who developed AKI compared with 100.5 $\pm 27.5 \mu \mathrm{mol} / \mathrm{L}$ in those patients who did not develop AKI $(P<$ $0.001)$. The increase in serum creatinine was sustained for at least 72 hours after CPB, suggesting the presence of intrinsic AKI rather than a prerenal aetiology. In Table 2, we present the concentration of urinary IL-18 comparing patients developing AKI with those who did not. Preoperatively, urinary IL-18 was virtually undetectable. There were no differences in urinary IL18 on arrival in ICU and at 24 hours postoperatively, even when adjusted for urinary creatinine concentration (Table 2). In Figures 1, 2, 3, 4, we show the AUC-ROCC for the prediction of AKI for urinary IL-18 and urinary IL-18/urinary creatinine ratio on arrival in ICU and at 24 hours, respectively. The predictive performance of urinary IL-18 was not better than chance at either time point (Figures 1 and 2), even after adjustment for urinary creatinine concentration (Figures 3 and 4). Even when using a variety of additional renal outcome measures in an exploratory analysis $>25 \%$ increase in creatinine, changes in creatinine to 72 hours or 5 days, sustained increase of greater than $50 \%$, sustained increase of greater than $25 \%$, and a variety of the RIFLE criteria [14]) or the serum creatinine criterion defining $\mathrm{AKI}$ according to the network classification [15], the AUC-ROCC for IL-18 was not greater than 0.61 (Tables $3,4,5,6$ ). Serum creatinine measured on arrival 
Table 2

Postoperative urinary interleukin-18 (IL-18) concentration

\begin{tabular}{|c|c|c|c|}
\hline Urinary IL-18 & $\operatorname{AKI}(n=20)$ & No $A K I(n=80)$ & $P$ value \\
\hline \multicolumn{4}{|l|}{ Unadjusted values } \\
\hline Preoperative, $\mathrm{pg} / \mathrm{mL}$ & $0(0-25)$ & $0(0-44)$ & 0.28 \\
\hline On arrival in the intensive care unit, $\mathrm{pg} / \mathrm{mL}$ & $168(19-736)$ & $104(36-292)$ & 0.70 \\
\hline At 24 hours postoperatively, $\mathrm{pg} / \mathrm{mL}$ & $195(54-537)$ & $165(42-288)$ & 0.47 \\
\hline \multicolumn{4}{|l|}{ Values adjusted for urinary creatinine } \\
\hline Preoperative, pg/mg & $0(0-12)$ & $0(0-32)$ & 0.32 \\
\hline On arrival in the intensive care unit, $\mathrm{pg} / \mathrm{mg}$ & $332(44-1,789)$ & $233(86-531)$ & 0.57 \\
\hline At 24 hours postoperatively, $\mathrm{pg} / \mathrm{mg}$ & $190(75-478)$ & $124(41-245)$ & 0.25 \\
\hline
\end{tabular}

Values denoting median and 25th to 75 th percentiles. $P$ values indicate Mann-Whitney $U$ test. AKI, acute kidney injury.

Table 3

Performance characteristics of urinary interleukin-18 (IL-18) according to different renal outcome definitions

\begin{tabular}{|c|c|c|}
\hline Variables & AUC-ROCC $(95 \% \mathrm{Cl})$ & $P$ value \\
\hline \multicolumn{3}{|c|}{ Increase in serum creatinine $>25 \%$ within 24 hours postoperatively } \\
\hline Urinary IL-18 on arrival in the intensive care unit & $0.58(0.46-0.71)$ & 0.19 \\
\hline Urinary IL-18 at 24 hours after cardiopulmonary bypass & $0.56(0.43-0.69)$ & 0.34 \\
\hline \multicolumn{3}{|c|}{ Increase in serum creatinine $>50 \%$ within 24 hours postoperatively } \\
\hline Urinary IL-18 on arrival in the intensive care unit & $0.56(0.38-0.74)$ & 0.51 \\
\hline Urinary IL-18 at 24 hours after cardiopulmonary bypass & $0.57(0.40-0.73)$ & 0.45 \\
\hline \multicolumn{3}{|c|}{ Increase in serum creatinine $>25 \%$ within 48 hours postoperatively } \\
\hline Urinary IL-18 on arrival in the intensive care unit & $0.59(0.47-0.71)$ & 0.14 \\
\hline Urinary IL-18 at 24 hours after cardiopulmonary bypass & $0.56(0.43-0.68)$ & 0.36 \\
\hline \multicolumn{3}{|c|}{ Increase in serum creatinine $>50 \%$ within 48 hours postoperatively } \\
\hline Urinary IL-18 on arrival in the intensive care unit & $0.53(0.38-0.68)$ & 0.70 \\
\hline Urinary IL-18 at 24 hours after cardiopulmonary bypass & $0.55(0.40-0.71)$ & 0.48 \\
\hline \multicolumn{3}{|c|}{ Increase in serum creatinine $>25 \%$ within 72 hours postoperatively } \\
\hline Urinary IL-18 on arrival in the intensive care unit & $0.60(0.39-0.71)$ & 0.63 \\
\hline Urinary IL-18 at 24 hours after cardiopulmonary bypass & $0.58(0.44-0.72)$ & 0.55 \\
\hline \multicolumn{3}{|c|}{ Increase in serum creatinine $>50 \%$ within 72 hours postoperatively } \\
\hline Urinary IL-18 on arrival in the intensive care unit & $0.58(0.36-0.66)$ & 0.85 \\
\hline Urinary IL-18 at 24 hours after cardiopulmonary bypass & $0.55(0.41-0.69)$ & 0.50 \\
\hline \multicolumn{3}{|c|}{ Increase in serum creatinine $>25 \%$ within 120 hours postoperatively } \\
\hline Urinary IL-18 on arrival in the intensive care unit & $0.60(0.48-0.72)$ & 0.10 \\
\hline Urinary IL-18 at 24 hours after cardiopulmonary bypass & $0.59(0.47-0.71)$ & 0.14 \\
\hline \multicolumn{3}{|c|}{ Increase in serum creatinine $>50 \%$ within 120 hours postoperatively } \\
\hline Urinary IL-18 on arrival in the intensive care unit & $0.52(0.38-0.67)$ & 0.78 \\
\hline Urinary IL-18 at 24 hours after cardiopulmonary bypass & $0.56(0.43-0.70)$ & 0.38 \\
\hline
\end{tabular}

AUC-ROCC, area under the curve for the receiver operating characteristic curve; $\mathrm{Cl}$, confidence interval. 
Table 4

Performance characteristics of urinary interleukin-18 (IL-18) according to different RIFLE criteria

\begin{tabular}{ll}
\hline Variables & AUC-ROCC (95\% Cl) \\
\hline RIFLE R or worse (R: $\mathrm{n}=31+\mathrm{I}: \mathrm{n}=13+\mathrm{F}: \mathrm{n}=6)$ & $0.61(0.48-0.74)$ \\
Urinary IL-18 on arrival in the intensive care unit & $0.57(0.43-0.71)$ \\
Urinary IL-18 at 24 hours after cardiopulmonary bypass & 0.12 \\
RIFLE I or worse (I: $\mathrm{n}=13+\mathrm{F}: \mathrm{n}=6)$ & $0.52(0.38-0.68)$ \\
Urinary IL-18 on arrival in the intensive care unit & $0.58(0.43-0.73)$ \\
Urinary IL-18 at 24 hours after cardiopulmonary bypass & 0.35 \\
\hline
\end{tabular}

For RIFLE classes [14], serum creatinine increases within 120 hours postoperatively and urinary output during the first 24 hours postoperatively. AUCROCC, area under the curve for the receiver operating characteristic curve; Cl, confidence interval; RIFLE, Risk of renal dysfunction, Injury to the kidney, Failure of kidney function, Loss of kidney function, and End-stage kidney disease; RIFLE F, RIFLE Failure; RIFLE I, RIFLE Injury; RIFLE R, RIFLE Risk.

in ICU had a higher value (AUC-ROCC 0.69, 95\% confidence interval 0.54 to $0.85 ; P=0.019$ ) to predict $\mathrm{AKI}$ (defined as a creatinine increase of greater than $50 \%$ within 48 hours postoperatively) than urinary IL-18 at that time of measurement. However, urinary IL-18 correlated with the duration of CPB (Spearman correlation coefficient $0.47 ; P<0.001$ ).

\section{Discussion}

In this prospective observational cohort study of 100 adult cardiac surgical patients, we analysed the predictive performance for AKI of early postoperative urinary IL-18 measurement on arrival in ICU and at 24 hours after commencement of CPB. In this setting, urinary IL-18 did not appear to be a useful biomarker for the prediction of AKI. AKI after CPB is associated with adverse hospital outcomes. Patients who develop AKI have increased morbidity and hospital mortality [1,2]. Early measurement of serum creatinine is considered to be of limited value in the prediction of AKI because it typically increases only when the glomerular filtration rate has decreased to less than $50 \%$ of normal $[7,15]$. Urinary IL-18, however, appears to have value in the prediction of AKI [6,10-13]. It has been demonstrated in renal tubular epithelial cells and in activated macrophages $[8,16]$. However, urinary IL-18, a promising renal biomarker for the early diagnosis of $\mathrm{AKI}$ in children undergoing cardiac surgery, has not yet been investigated in adult cardiac surgical patients.
The good predictive performance of urinary IL-18 for the diagnosis of $\mathrm{AKI}$ in a recent study of paediatric cardiac surgical patients [13] was not confirmed for adults in this study. However, in paediatric cardiac surgical patients, comorbidities do not play such a major and confounding role compared with adults. Adult cardiac surgical patients often present with agerelated comorbidities and with diminished renal reserve. Accordingly, observations made in paediatric populations may not apply to adult patients. This appears to be the case for urinary IL-18.

Our negative findings are in accordance with a previous study investigating the value of urinary IL-18 in the prediction of AKI following exposure to contrast for coronary intervention in adult patients [9]. While it is conceivable that the very early peak urinary IL-18 concentration was missed in this study [9] - even when measured (as we did) at an early stage ( 6 hours after the injurious event to the kidney commenced) - urinary IL18 was not able to predict AKI. It is nonetheless important to note that patients undergoing CPB who did not develop AKI had elevated postoperative urinary IL-18 concentrations compared with preoperative values. There are at least three possible explanations: (a) all patients undergoing CPB suffer mild $\mathrm{AKI}$, which resolves quickly and does not lead to any elevation in serum creatinine; or (b) urinary IL-18 increases independently of kidney injury, due to nonrenal factors such as systemic inflammation; or (c) both explanations apply. Indeed, we found

Table 5

\section{Performance characteristics of urinary interleukin-18 (IL-18) according to different network criteria of acute kidney injury}

\begin{tabular}{lr}
\hline Variables & AUC-ROCC (95\% Cl) \\
\hline AKIN stage 1 or worse $(\mathrm{n}=32)$ & $0.48(0.35-0.61)$ \\
$\quad$ Urinary IL-18 on arrival in the intensive care unit & 0.80 \\
Urinary IL-18 at 24 hours after cardiopulmonary bypass & $0.15 \quad 0.46-0.72)$ \\
\hline
\end{tabular}

Acute kidney injury defined according to the network criteria [15] with serum creatinine increase of greater than $26.4 \mu \mathrm{mol} / \mathrm{L}$ or greater than $0.3 \mathrm{mg} / \mathrm{dL}$ within 48 hours postoperatively. AKIN, Acute Kidney Injury Network; AUC-ROCC, area under the curve for the receiver operating characteristic curve; Cl, confidence interval. 
Performance characteristics of urinary interleukin-18 (IL-18) to predict the development of sustained acute kidney injury

\begin{tabular}{|c|c|c|}
\hline Variables & AUC-ROCC $(95 \% \mathrm{Cl})$ & $P$ value \\
\hline \multicolumn{3}{|c|}{ Increase in serum creatinine $>25 \%$ within 48 hours postoperatively } \\
\hline Urinary IL-18 on arrival in the intensive care unit & $0.58(0.46-0.71)$ & 0.19 \\
\hline Urinary IL-18 at 24 hours after cardiopulmonary bypass & $0.56(0.43-0.69)$ & 0.43 \\
\hline \multicolumn{3}{|c|}{ Increase in serum creatinine $>50 \%$ within 48 hours postoperatively } \\
\hline Urinary IL-18 on arrival in the intensive care unit & $0.56(0.38-0.74)$ & 0.51 \\
\hline Urinary IL-18 at 24 hours after cardiopulmonary bypass & $0.57(0.40-0.73)$ & 0.45 \\
\hline \multicolumn{3}{|c|}{ Increase in serum creatinine $>25 \%$ within 120 hours postoperatively } \\
\hline Urinary IL-18 on arrival in the intensive care unit & $0.59(0.47-0.71)$ & 0.14 \\
\hline Urinary IL-18 at 24 hours after cardiopulmonary bypass & $0.56(0.43-0.68)$ & 0.36 \\
\hline \multicolumn{3}{|c|}{ Increase in serum creatinine $>50 \%$ within 120 hours postoperatively } \\
\hline Urinary IL-18 on arrival in the intensive care unit & $0.51(0.34-0.67)$ & 0.93 \\
\hline Urinary IL-18 at 24 hours after cardiopulmonary bypass & $0.57(0.42-0.73)$ & 0.36 \\
\hline \multicolumn{3}{|l|}{ RIFLE R or worse $(R: n=31+I: n=13+F: n=6)$} \\
\hline Urinary IL-18 on arrival in the intensive care unit & $0.61(0.48-0.74)$ & 0.12 \\
\hline Urinary IL-18 at 24 hours after cardiopulmonary bypass & $0.57(0.43-0.71)$ & 0.29 \\
\hline \multicolumn{3}{|l|}{ RIFLE I or worse $(I: n=13+F: n=6)$} \\
\hline Urinary IL-18 on arrival in the intensive care unit & $0.52(0.37-0.67)$ & 0.30 \\
\hline Urinary IL-18 at 24 hours after cardiopulmonary bypass & $0.58(0.43-0.73)$ & 0.28 \\
\hline
\end{tabular}

Acute kidney injury is defined as at least two consecutive significantly increased serum creatinine values. For RIFLE classes, serum creatinine increases within 120 hours postoperatively and urinary output during the first 24 hours postoperatively. AUC-ROCC, area under the curve for the receiver operating characteristic curve; Cl, confidence interval; RIFLE, Risk of renal dysfunction, Injury to the kidney, Failure of kidney function, Loss of kidney function, and End-stage kidney disease; RIFLE F, RIFLE Failure; RIFLE I, RIFLE Injury; RIFLE R, RIFLE Risk.

that duration of CPB correlated with postoperative urinary IL18. On the basis of our findings, increases in urinary IL-18 may indicate renal epithelial tubular cell injury in adult cardiac surgical patients, which is not severe enough to predictably lead to loss of glomerular filtration rate. Thus, urinary IL-18 is potentially a nonspecific marker of inflammation and not a specific marker for AKI. Indeed, IL-18 is released by both renal tubular cells and macrophages or blood monocytes and is then filtered through the glomerular basal membrane. The latter source, however, is believed to represent the main origin of IL18 in adults [8]. If this were true, it would potentially confound the value of urinary IL-18 as a specific renal biomarker and at least partly explain our observations. In children, release of IL18 may proceed primarily through the kidney rather than immune cells, thus explaining the better predictive ability of IL18. Irrespective of the possible explanation, our observations highlight the need to study the value of renal biomarkers in adult cardiac surgical patients separately.

This is a single-centre study and its findings need to be confirmed or refuted in other centres. While it is conceivable that a larger sample size or more frequent measurement might have generated statistically significant findings on urinary IL-18, the study sample size is sufficient for the detection of a major and clinically relevant predictive performance of this renal biomarker. Although we enrolled a patient population with a typical mix of cardiac surgical procedures, it is possible that urinary IL18 would be of greater value in patients at increased risk of developing AKI. In the absence of adjudication of aetiology, the sustained increase in serum creatinine may exclude, at least in part, haemodynamic/prerenal causes of AKI, for which no increase in urinary IL-18 would be expected. Also, worse outcome in patients developing AKI (as defined) compared with those who did not develop AKI points to intrinsic rather than prerenal azotaemia. We consider that additional novel biomarkers with increased predictive performance are still needed for more precise and earlier prediction of AKI after adult cardiac surgery.

\section{Conclusion}

This is the first study to test the value of urinary IL-18 as a predictor of AKI following adult cardiac surgery with CPB. Our findings suggest that, although urinary IL-18 may be a marker of CPB-associated inflammation, it is not useful in identifying patients who will subsequently develop AKI after adult cardiac surgery. 


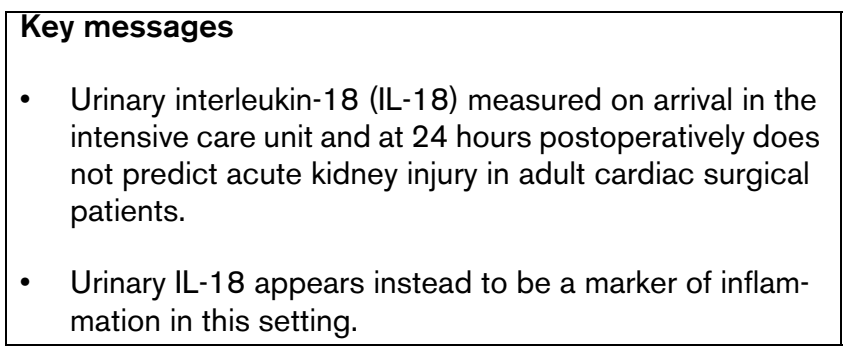

\section{Competing interests}

The authors declare that they have no competing interests.

\section{Authors' contributions}

$\mathrm{MH}$ and $\mathrm{AH}-\mathrm{F}$ participated in the design of the study, performance of the statistical analysis, and drafting of the manuscript. $\mathrm{RB}$ conceived of the study and participated in the design of the study, performance of the statistical analysis, and drafting of the manuscript. PD carried out the urinary IL-18 measurements. DS participated in the design and coordination of the study and helped to draft the manuscript. All authors read and approved the final manuscript.

\section{Acknowledgements}

$\mathrm{MH}$ holds a postdoctoral Feodor-Lynen Research Fellowship from the Alexander von Humboldt-Foundation, Germany. This study was partly funded by a grant from the Australian and New Zealand College of Anaesthetists and by the Austin Hospital Anaesthesia and Intensive Care Trust Fund.

\section{References}

1. Ostermann ME, Taube D, Morgan CJ, Evans TW: Acute renal failure following cardiopulmonary bypass: a changing picture. Intensive Care Med 2000, 26:565-571.

2. Uchino S, Kellum JA, Bellomo R, Doig GS, Morimatsu H, Morgera S, Schetz M, Tan I, Bouman C, Macedo E, Gibney N, Tolwani A, Ronco C, Beginning and Ending Supportive Therapy for the Kidney (BEST Kidney) Investigators: Acute renal failure in critically ill patients: a multinational, multicenter study. JAMA 2005, 294:813-818.

3. Haase M, Haase-Fielitz A, Bagshaw SM, Reade MC, Morgera S, Seevenayagam S, Matalanis G, Buxton B, Doolan L, Bellomo R: Phase II, randomized, controlled trial of high-dose $\mathrm{N}$-acetylcysteine in high-risk cardiac surgical patients. Crit Care Med 2007, 35:1324-1331.

4. Molitoris BA: Transitioning to therapy in ischemic acute renal failure. J Am Soc Nephrol 2003, 14:265-267.

5. Parikh CR, Abraham E, Ancukiewicz M, Edelstein CL: Urine IL-18 is an early diagnostic marker for acute kidney injury and predicts mortality in the intensive care unit. J Am Soc Nephrol 2005, 16:3046-3052.

6. Parikh CR, Jani A, Melnikov VY, Faubel S, Edelstein CL: Urinary interleukin-18 is a marker of human acute tubular necrosis. Am J Kidney Dis 2004, 43:405-414.

7. Vaidya VS, Ramirez V, Ichimura T, Bobadilla NA, Bonventre JV: Urinary kidney injury molecule-1: a sensitive quantitative biomarker for early detection of kidney tubular injury. Am J Physiol Renal Physiol 2006, 290:F51 7-F529.

8. Obregon C, Dreher D, Kok M, Cochand L, Kiama GS, Nicod LP: Human alveolar macrophages infected by virulent bacteria expressing SipB are a major source of active interleukin-18. Infect Immun 2003, 71:4382.

9. Bulent Gul C, Gullulu M, Oral B, Aydinlar A, Oz O, Budak F, Yilmaz $Y$, Yurtkuran M: Urinary IL-18: a marker of contrast-induced nephropathy following percutaneous coronary intervention? Clin Biochem 2008, 41:544-547.
10. Ling $W$, Zhaohui $N$, Ben $H$, Leyi G, Jianping L, Huili D, Jiaqi Q: Urinary IL-18 and NGAL as early predictive biomarkers in contrast-induced nephropathy after coronary angiography. Nephron Clin Pract 2008, 108:c176-c181.

11. Parikh CR, Jani A, Mishra J, Ma Q, Kelly, Barasch J, Edelstein CL, Devarajan P: Urine NGAL and IL-18 are predictive biomarkers for delayed graft function following kidney transplantation. $\mathrm{Am}$ J Transplant 2006, 6:1639-1645.

12. Washburn KK, Zappitelli M, Arikan AA, Loftis L, Yalavarthy $R$, Parikh CR, Edelstein CL, Goldstein SL: Urinary interleukin-18 is an acute kidney injury biomarker in critically ill children. Nephrol Dial Transplant 2008, 23:566-572.

13. Parikh CR, Mishra J, Thiessen-Philbrook H, Dursun B, Ma Q, Kelly C, Dent C, Devarajan P, Edelstein CL: Urinary IL-18 is an early predictive biomarker of acute kidney injury after cardiac surgery. Kidney Int 2006, 70:199-203.

14. Bellomo R, Kellum JA, Ronco C: Defining and classifying acute renal failure: from advocacy to consensus and validation of the RIFLE criteria. Intensive Care Med 2007, 33:409-413.

15. Herget-Rosenthal S, Marggraf G, Hüsing J, Göring F, Pietruck F, Janssen O, Philipp T, Kribben A: Early detection of acute renal failure by serum cystatin C. Kidney Int 2004, 66:1115-1122.

16. Faust J, Menke J, Kriegsmann J, Kelley VR, Mayet WJ, Galle PR, Schwarting A: Correlation of renal tubular epithelial cellderived interleukin-18 up-regulation with disease activity in MRL-Faslpr mice with autoimmune lupus nephritis. Arthritis Rheum 2002, 46:3083-3095. 